\title{
Agent-based modelling as a research tool for criminological research
}

\author{
Charlotte Gerritsen
}

\begin{abstract}
Computational modelling techniques, originating from fields like Computer Science and Artificial Intelligence, may be beneficial for criminological research. Because of their formal nature, computational models can be processed by machines that operate on them, for example for the purpose of simulation. As a consequence, these techniques may help gain insights that lacked based of purely informal theories. A well-known example of such a technique, which has become widely applied within criminology, is called agent-based modelling.

Agent-based modelling (ABM) is a computational method that enables a researcher to create, analyse and experiment with models composed of agents, i.e., autonomous pieces of software that interact within a computational environment (Gilbert, 2008). In the current article this technique will be explored in depth. First, I will give a description of the technique and present the architecture of an ABM. Subsequently, I will apply the technique to a simple toy example in the context of a simulation model of the bystander effect, to demonstrate the possibilities of the approach. I will discuss some pros and cons of the approach and present related work to help appreciate the benefits of applying ABM to different criminological research questions. Hopefully, this will provide readers with the necessary knowledge to consider the use of ABM in their own research.
\end{abstract}

Keywords: Agent-based modelling; Simulation; Research method; Criminology; Bystander effect

\section{Background}

Within the field of Criminology, many theories exist about various (biological, psychological and social) aspects of deviant behaviour. However such theories in the criminological domain are often informal i.e. not in a computational format. This is not a problem if one wants to understand what is happening on an abstract level. However, when detailed dynamics are studied, it is important to take the influence of all aspects (and their interaction) into account. This can still be done by using an informal theory, but when the number of aspects increases it will be more difficult to oversee all dynamic patterns that result from the interaction between these aspects. Thus when the numbers increase, it can be very helpful to represent the theories in a computational format, in such a way that they can be used to perform simulation. This will make it possible to perform automated (pseudo-) experiments, in order to gain more insight in a phenomenon, and possibly refine the original theory.

Correspondence: CGerritsen@nscr.nl

Netherlands Institute for the Study of Crime and Law Enforcement, Amsterdam, Netherlands
In the last decades, computational modelling has found its way to the field of Criminology. When investigating the literature on computational modelling of displacement of crime, a wide variety of different computational modelling approaches can be distinguished (Gerritsen and Bosse 2015). Among the approaches that are applied, one can find agent-based modelling (Gilbert 2008; Bosse and Gerritsen 2010; Bosse et al. 2011; Brantingham et al. 2005; Reis et al. 2006), population-based modelling (Bosse et al. 2011), cellular automata (Hayslett-McCall et al. 2008; Liu et al. 2005), different spatial analysis techniques (Groff 2005), and evolutionary computing techniques (Reis et al. 2006).

The underlying principle behind agent-based modelling approaches is the 'agent' metaphor, i.e., the idea to compose a model of autonomous pieces of software that make their own decisions, based on information they observe in their direct environment. For instance, the agent-based approach presented in Bosse and Gerritsen, (2010) simulates the spatio-temporal dynamics of crime as a result of individual decisions of offenders, targets, and guardians (e.g., to move around, or to perform assaults or arrests). In contrast, population-based modelling

\section{实}

() 2015 Gerritsen; licensee Springer. This is an Open Access article distributed under the terms of the Creative Commons Attribution License (http://creativecommons.org/licenses/by/4.0), which permits unrestricted use, distribution, and reproduction in any medium, provided the original work is properly credited. 
approaches (e.g., Bosse et al. 2011) do not distinguish individual agents, but instead describe the dynamics of crime in terms of mathematical formulae (mostly differential equations) over variables that represent the densities of certain sub-groups in the population. Furthermore, Cellular Automata (CA) are discrete models that consist of grids of cells that are in particular states (e.g., on or off), of which the dynamics are determined by rules that take the states of adjacent cells into account. Liu et al. (2005) have used CAs to simulate individual crime events, in order to generate plausible crime patterns. In their approach, the main elements are offenders, targets, and crime places, of which different attributes can be manipulated, such as motivation of offenders, capability of guardians, and accessibility of places. Additionally, a number of spatial analysis techniques are used in Gerritsen (2010); these techniques include, among others, Geographical Information Systems (GIS) and analytical methods. Finally, evolutionary computing is a sub-area of Artificial Intelligence that attempts to find optimal solutions to mathematical problems by exploiting a computational variant of biological evolution. More specifically, by representing candidate solutions to an optimisation problem in terms of individuals in a population, and having the population evolve using operations such as recombination and mutation (where the better performing individuals have a higher probability to reproduce), good solutions can be found to problems in a variety of domains, including criminology. For example, in Reis et al. (2006) some results are presented that were achieved with GAPatrol, an evolutionary multi agent-based simulation tool devised to assist police managers in the design of effective police patrol route strategies.

In this article I will explore the agent-based modelling technique in depth.

First, I will give a description of the technique and present the architecture of an ABM. Subsequently, I will apply the technique to a simple example in the context of a simulation model of the bystander effect, to demonstrate the possibilities of the approach. I will discuss some pros and cons of the approach and present related work to help appreciate the benefits of applying ABM to different criminological research questions. Hopefully, this will provide readers with the necessary knowledge to consider the use of ABM in their own research.

\section{Agent-based modelling}

As mentioned in the introduction, agent-based modelling is a computational method that enables the researcher to create, analyse and experiment with models composed of agents. Here, agents are autonomous (software) entities that interact with each other and with their environment in some artificial (simulated) world (Gilbert 2008). Hence, ABM is a specific type of modelling, where the real world system that is modelled consists of (one or multiple) autonomous entities (e.g., human beings, animals, or intelligent artefacts). According to Shannon (1975), a model is a representation of an object, system or idea other than that of the entity itself. In a model the most important concepts of the relevant real world system are described, as well as the relationships between them. The subject of the model may be very complex (e.g., the spatio-temporal dynamics of burglary in a city) and it is often the case that not all concepts or relationships are known. This means that it is usually not possible to describe all aspects and relations completely and unambiguously. Hence, a model is typically a simplification of reality and is not presumed to be a complete representation. Further, a model is based on choices and assumptions made by the designer; characteristics of a model are not necessarily characteristics of reality and this implies that conclusions that are drawn based on the model are based on the assumptions made by the designer.

Although this does not hold for all models, it is often useful if a model can imitate the dynamics of a process over time, in order to accurately study a model. This process is called simulation and models that can be used to simulate behaviour over time are called dynamical models. By imitating the dynamics of a process over time, a simulation model helps to clarify the interaction between different aspects. The outcomes of a simulation are usually represented in a graphical form and depict a sequence of states of the model at subsequent time points (simulation run or trace).

Models are usually used as a convenient manner to study real world processes. Studying a model instead of a process itself has a number of advantages. For instance, it is possible to study a model of a process that does not yet exist in real life. An example is the impact of installing surveillance cameras at a particular location on the behaviour of citizens. Think about installing cameras in an entertainment area, or traffic cameras near the highway. To determine where and how many of the cameras should be placed, it is useful to test the effect of these (hypothetical) interventions in advance. Another advantage of studying a model is the option to study a process that cannot be studied directly. An example of such a process is a cognitive process, such as human decision making. In this case, it is not always trivial to get insight in the mechanisms that are going on in a person's mind. In both situations a model may provide the researcher more insight in the process under investigation, even it is not completely identical to the world. Another reason often mentioned is prediction; in that case, models are used to see what will probably happen in the 'real' future (as opposed to the 'hypothetical' future, as was the case with the camera example); this applies, for instance, to the case of economical or weather models. Finally, it can be cheaper, less time consuming, and more 
feasible to perform experiments using a model than in the real world.

Also in the existing literature on agent-based modelling, the underlying reasons used for building the models differ. For example, some authors try to develop simulation models of crime displacement in existing cities, which can be directly related to real world data (e.g., Liu et al. 2005), for instance to make predictions, whereas others deliberately abstract from empirical information (e.g., Bosse et al. 2011). The idea behind the latter perspective is that the simulation environment is used as an analytical tool, mainly used by researchers and policy makers, for thought experiments, to shed more light on the process under investigation, and perhaps improve existing policies (e.g., for surveillance) (Elffers and Van Baal 2008). Also, some authors take an intermediate point of view (e.g., Bosse and Gerritsen 2010; Malleson and Brantingham 2008). They initially build their simulation model to study the phenomenon per se, but define its basic concepts in such a way that it can be directly connected to empirical information, if this becomes available.

\section{The process of modelling and simulation}

When one wants to develop a model and perform simulations, two phases can be distinguished. The first phase is the design phase. In the design phase the model is built. The phase consists of the conceptualisation and formalisation of a process. Once the model has been designed, it can be analysed in the second phase. This phase consists of the simulation of the process and the evaluation of the model. I will explain the complete process in this section.

\section{Step 1: Conceptualisation}

The first important decision when designing a model is choosing what exactly one wants to model. This means that the modeller should know which process needs to be simulated and which questions (s)he wants to be answered by using the model. When this has been decided, the next step is the conceptualisation of the process. During this step the relevant concepts need to be identified. Which factors play a role? A concept can be an object (e.g., a house or a person) but might be an event as well (e.g., a robbery). The second task is specifying the relationships between the concepts. It is specified whether different concepts influence each other, but not yet how they influence each other. The results of specifying the relationships between concepts can be a list of statements in the form 'A affects B', where it is possible that concepts are influenced by multiple concepts and concepts influence multiple concepts. The relationships can be represented in a graph as well. Then, the concepts should be depicted as nodes (circles) and the relationships as edges (arrows) between the nodes (see Figure 1).

\section{Step 2: Formalisation}

After the model has been conceptualised, the next step is its formalisation. During this step, the concepts and relations between concepts are defined in more detail, using some machine-readable modelling language. Two approaches that are often considered are logic-oriented approaches and mathematical approaches (based on difference or differential equations) (Bosse et al. 2007). Logicoriented approaches are good for expressing qualitative relations, while mathematical approaches are especially suitable for quantitative relations.

In mathematical modelling approaches, concepts are represented by variables with a value. Relations are rules that specify how the value of one variable can be calculated from the values of other variables. For each concept, variables are defined that can have certain values. Establishing the relations between the concepts requires careful thinking. The influence can take several forms, depending on the values that a variable can have and on the time delay related to the effect. For example, a concept $A_{2}$ that is affected by another concept $A_{1}$ in a next time step can be specified as follows:

$$
A_{2}(t+\Delta t)=A_{2}(t)+f\left(A_{1}(t), A_{2}(t)\right) \Delta t
$$

In (1), $t$ is a variable representing the time, $\Delta t$ (delta $t$ ) stands for a change in the time variable, and $f$ is some function. Similarly, multiple concepts affecting a given concept can be modelled.

In case a logical modelling approach is used, concepts and their properties are represented by statements about the world that can be true or false (sometimes called state properties). For example, a statement could be robbery (P1,P2), which could represent the concept that it is true that person $\mathrm{P} 1$ robs person P2. Temporal relations between concepts are rules that specify statements such that

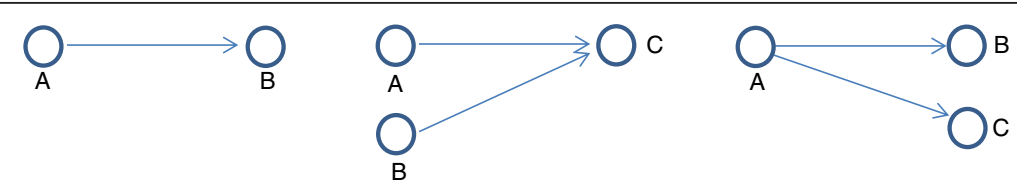

Figure 1 A: one concept influences one other concept; B: two concepts influence one concept; C: one concept influences two other concepts. 
when at some time point they are true then another statement will be true at another (next) time point. For example, in the LEADSTO language (Bosse et al. 2007), which has previously been used to model a number of criminological processes (Gerritsen 2010), the temporal relation $\mathrm{A} \rightarrow \mathrm{B}$ denotes that when a state property $\mathrm{A}$ occurs, then after a certain time delay (which for each relation instance can be specified as any positive real number), state property B will occur. These rules can combine statements in several ways, using logical operators. A few combinations that are often used, are listed below in Table 1 (note that for simplicity characters like A, B, etcetera, are used instead of statements of the form "robbery (P1,P2)".

Please note that LEADSTO is only one of many modelling languages that can be used. For an elaborate survey of approaches, such as Netlogo (Banos et al. 2015), MASON (Luke et al. 2005) and JADE (Bellifemine et al. 2007) please see (Nikolai and Madey 2009). More details about LEADSTO are available in Bosse et al. (2007).

\section{Step 3: Simulation}

To perform a simulation experiment, first the question or the pattern to be addressed needs to be formulated. Setting up a simulation experiment requires a structured plan with respect to the values chosen for the variables and parameters, thereby representing a certain scenario. So as a next step, choices need to be made with respect to the initial values of variables and the values of the parameters involved in the simulation model. The setting of these values is determined by the characteristics of the process under investigation (for instance, the number of houses in a city, or the ratio between citizens and guardians can be inspired by statistics on the actual situation). However, it is also possible to set the parameters by using fictive information. Different scenarios can be set up, in order to compare the outcomes for different circumstances, which can be accomplished by changing the values in a number of different simulations. For a thorough evaluation, multiple scenarios need to be simulated. Some models contain a stochastic element, i.e., parameters for which the values are determined by a

Table 1 Some examples of logical relations

\begin{tabular}{|c|c|}
\hline Logical relation & Meaning \\
\hline$A \wedge B \rightarrow C$ & if $A$ is true and $B$ is true, then $C$ will be true \\
\hline$A \vee B \rightarrow C$ & $\begin{array}{l}\text { if } A \text { is true, } B \text { is true, or both are true, then } C \text { will } \\
\text { be true }\end{array}$ \\
\hline$A(X) \rightarrow B(X)$ & $\begin{array}{l}\text { if } A \text { is true for a specific value of } X \text {, then } B \text { will be } \\
\text { true for that value of } X\end{array}$ \\
\hline $\operatorname{not}(A) \rightarrow B$ & if $A$ is not true, then $B$ will be true \\
\hline$A(X) \wedge X>52 \rightarrow B$ & $\begin{array}{l}\text { if } A \text { is true for a specific value of } X \text {, which is larger } \\
\text { than } 52 \text {, then } B \text { will be true }\end{array}$ \\
\hline
\end{tabular}

probability. With these kind of models, running the same model several times results in possibly different traces, which all reflect the outcome of a different simulation for the same model.

\section{Step 4: Evaluation}

The final phase of the modelling and simulation cycle is the evaluation phase. In this phase it is verified whether or not the model is a correct representation of the system that it represents. This can be done by formulating certain properties that hold in the actual situation and check whether they also hold in the model. These properties may be derived either from theory (in this case one speaks about internal validation) or from empirical data (external validation). Such properties often express higher-level characteristics of the behaviour of the process rather than the direct influences; for example stable end situations (e.g., 'after some time the crime rate stabilises'), or the effect of the occurrence of several events at the same time. A distinction can be made between quantitative properties, which are statements about numerical characteristics of the model, and qualitative properties, which are statements about non-numerical relations or characteristics of the model. Properties of a simulation model can be checked automatically by various computer programs.

When the evaluation shows that the model does not resemble the real process well enough, there may be three possible causes, namely, 1) a modelling flaw, 2) wrongly chosen values for the parameters that describe the influence from one concept on another, or 3) an invalid theory that was used as a basis for the model. In case of 1) and 2 ), the behaviour of the model is not as expected by the modeller. For instance, in case of 1 ), the modeller might have intended to reproduce the phenomenon that crime takes place whenever a motivated offender meets a suitable target, but when executing the simulation this turns out not to happen. In case of 2), the situation is slightly different. In this case, for instance, the simulation might indeed show that crime takes place, but the frequency or timing of the criminal activities may be incorrect. Finally, in case of 3), the behaviour of the model is indeed as expected by the modeller, but when executing the simulation it turns out that this behaviour is not realistic. For instance, the assumption that crime always takes place when a motivated offender meets a suitable target might turn out to be too simplistic; hence the underlying theory would need to be refined.

Based on the type of error, different approaches need to be taken to deal with the problem. In the first case, the modelling cycle will continue with a re-conceptualisation phase. In the second case, the parameters need to be chosen differently. This can be done heuristically, by trying different values that results in a more realistic simulation 
or by mathematical approaches that find optimal parameter values (also known as parameter tuning). To apply parameter tuning, realistic data about the simulated process is required. The tuning process then finds parameter values that, when used in the model, achieve results that are as close as possible to the empirical data. In the third situation (i.e., there is a discrepancy between the theory used as a basis for the model and the phenomena observed in the real world), the model can be used as a tool to find out where the theory is incorrect (e.g., by comparing simulation results with empirical data). The result of this step is usually that the theory itself is altered or refined.

When the evaluation gives a satisfactory result, the validated model can be used as a tool for studying the process that has been described. Different hypothetical scenarios can be simulated and compared to improve the understanding of the process or evaluate the effect of changes in the scenario.

\section{The bystander effect: agent-based simulation of people's reaction to norm violation}

In this section the modelling and simulation cycle is applied to a concrete example: the bystander effect. This term applies to cases when people base their decisions on whether or not to intervene in some situation (usually regarding some incident or norm violation by other people) on the presence of bystanders. For example, imagine you see someone falling on the ground. On the one hand, this could be caused by a heart attack (in which case it is probably a good idea to help), but on the other hand, the person may also have tripped over his shoelace (in which case he will easily be able to stand up by himself). The question is then: do you decide to help or not?

Research by (Latané and Darley 1968, Latané and Nida 1981) about these kinds of emergency situations demonstrated that the mere presence of other people decreases our willingness to help victims. Other studies by Freeman et al. (1975) and Levy et al. (1972) demonstrated that the same effect also applies to cases of norm violation (for instance, when you walk down the street and see two people fighting).

An important factor in such cases is that people do not have sufficient information to decide how they should act. In principle, people are not unwilling to help, but they do not know if their interpretation of the situation is correct. As a result, they typically seek for confirmation by observing the behaviour of other bystanders; hence their behaviour is largely determined by social cognition.

According to Latané and Nida (1981), three social psychological processes might be used to explain why the presence of other people affects the decision to intervene, namely audience inhibition (people run the risk of embarrassment if they decide to intervene while they misinterpreted the situation), social influence (people confirm to the behaviour of others) and diffusion of responsibility (reducing the psychological costs of nonintervention: why would I help while all other bystanders are also capable but do not do a thing?).

Below, an agent-based model of the bystander effect will be generated by following the four steps of the modelling and simulation methodology introduced earlier. By generating such a model, the effect of bystanders on intervention behaviour regarding norm violations can be studied. This enables the researcher to gain more insight in situations such as, e.g., 'what happens if person $\mathrm{P}$ is confronted with event $\mathrm{E}$, in a situation with $\mathrm{N}$ bystanders?'.

\section{Step 1: Conceptualisation}

As mentioned above, the first step one needs to apply when developing a computational model is to make a list of concepts that are relevant for the process to be modelled. In case of the bystander effect, we restrict ourselves to the decision making behaviour of one individual (i.e., the person that will deliberate whether or not to intervene in some situation). To model decision making processes, an often used approach is the BDI-model (Rao and Georgeff 1991), which explains the reasoning process people undertake to get to certain actions in terms of $B$ (eliefs), D(esires), and I(ntentions). It typically states that people first have certain desires, then generate certain intentions to fulfil these desires (assuming that certain other criteria are true as well, such as the belief that this intention contributes to the desire), and finally they realise their intentions in terms of actions. Hence, using this model already gives some structure for the decision making process to be modelled. Next, a number of additional beliefs have been introduced, inspired by the theories on the bystander effect explained above. For instance, we need beliefs about the seriousness of the situation, the presence and capabilities of other people, the person's own capabilities, and so on. Finally, some observations and actions are needed, to model the interaction between the person and its environment. A graphical overview of all concepts and the relationships between them is provided in Figure 2. An informal explanation of all concepts is given in Table 2. Roughly, this model states that the person generates a desire to intervene if (s)he beliefs that there really is an emergency, i.e. if the observed characteristics of the situation are more serious than the person's individual 'norm' for intervention. In addition, if the person also believes that (s)he personally has the responsibility to intervene (because the costs for intervention are not too high, and the inhibition of the audience is not strong), then (s)he generates the intention to intervene. Finally, if the person also believes that (s)he is capable of helping, than the actual intervention is executed. More details are provided in the next section. 


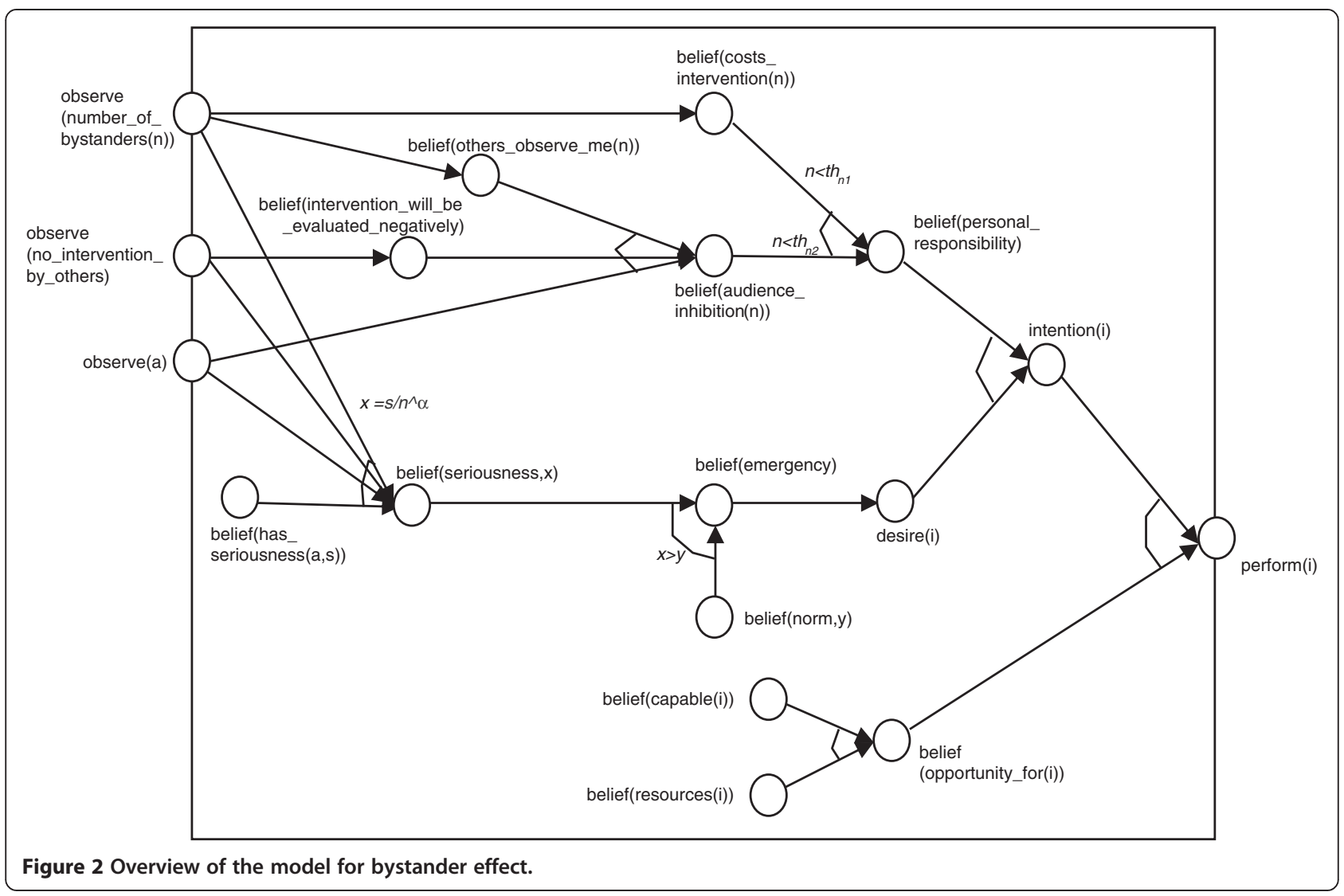

Table 2 Overview of states properties used in the model

\begin{tabular}{ll}
\hline Formal state property & Description \\
\hline observe(number_of_bystanders(n)) & The agent observes $n$ bystanders \\
observe(no_intervention_by_others) & The agent observes that the other bystanders do not intervene \\
observe(a) & The agent observes that action a is performed \\
belief(costs_intervention(n)) & The agent believes that the costs of intervention are $n$ \\
belief(others_observe_me(n)) & The agent believes that $n$ others observe him \\
belief(intervention_will_be_evaluated_negatively) & The agent believes that intervention will be evaluated negatively \\
belief(audience_inhibition(n)) & The agent believes there is an audience inhibition (i.e. risk of misinterpretation) of level $n$ \\
belief(personal_responsibility) & The agent believes he is personally responsible for intervention \\
belief(has_seriousness(a,xi)) & The agent believes that action a has a seriousness of level x1 \\
belief(seriousness, $x 2)$ & The agent believes that the current situation has a seriousness of level x2 \\
belief(norm,y) & The agent believes his personal norm for seriousness has level y \\
belief(emergency) & The agent believes there is an emergency situation \\
belief(capable(i)) & The agent believes he is capable of intervention \\
belief(resources(i)) & The agent believes he has the resources for intervention \\
belief(opportunity(i)) & The agent believes there is an opportunity for intervention \\
desire(i) & The agent has the desire to intervene \\
intention(i) & The agent has the intention to intervene \\
perform(i) & The agent performs an intervention \\
\hline
\end{tabular}




\section{Step 2: Formalisation}

To formalise the dynamics of the bystander model, the LEADSTO language introduced above was used (Bosse et al. 2007). As mentioned earlier, this language enables the modeller to specify the influences between concepts in a formal manner. In particular, for each arrow or combination of arrows (denotes by an arc) in Figure 2, a LEADSTO rule has been introduced. The complete set of 11 rules, together with an information explanation, can be found below. Several types of rules are distinguished, namely rules 1) to determine the belief that the agent is personally responsible, 2) to determine the desire to help, 3) to determine the belief that there is an opportunity to help and 4) to perform the action. Note that the $\mathrm{x}$ symbols indicate the 'type' of variables that are involved (for instance, a real or natural number, or an action to intervene).

\section{1) Personal responsibility}

R1 "If you observe that others do not intervene this leads to the belief that intervention will be evaluated negatively (social influence)"

observe(no_intervention_by_others) $\rightarrow \rightarrow$

belief(intervention_will_be_evaluated_negatively)

R2 "If you observe a number of bystanders present then you will believe that the bystanders can observe you"

\section{$\forall \mathrm{n}: I N T E G E R$}

observe(number_of_bystanders(n)) $\rightarrow \rightarrow$

belief(others_observe_me(n))

R3 "If you observe an action and you believe that others can observe you and that intervention will be evaluated negatively this will lead to the belief of audience inhibition with value n"

\section{$\forall a: A C T I O N \forall$ n:INTEGER}

observe $(a) \wedge$ belief(others_observe_me $(n)) \wedge$

belief(intervention_will_be_evaluated_negatively) $\rightarrow \rightarrow$ belief(audience_inhibition(n))

R4 "The number of bystanders that you observe determines your belief about the costs of intervention. The higher the number of bystanders the higher the costs (diffusion of responsibility)"

\section{$\forall \mathrm{n}:$ INTEGER}

observe(number_of_bystanders(n)) $\rightarrow \rightarrow$

belief(costs_intervention(n))
R5 "Your belief about the costs of intervention combined with your belief of audience inhibition determines your belief on personal responsibility. The lower the costs and audience inhibition the higher the belief of personal responsibility"

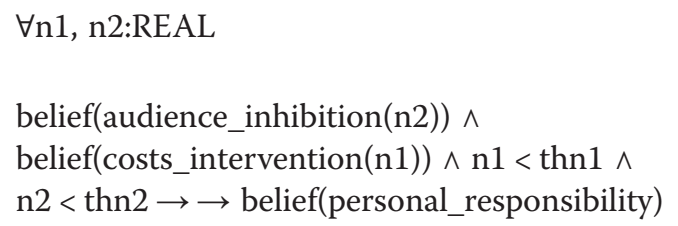

The thresholds used in R5 (thn1 and thn2) are linked to the level of seriousness. If you believe that a certain violation is very serious, and you feel highly personally implicated then you will probably less likely let the costs of intervention or audience inhibition stop you from intervening. The threshold for nonintervention is high. However, if you believe a violation is not serious at all, then you will let the costs and audience inhibition keep you from intervening. In this case the threshold for non-intervention will be low.

\section{2) Desire to help}

R6 "When you observe an action and you believe that this action has a seriousness of value $s$ and you see that there are bystanders present and that these bystanders do not intervene then this leads to the belief that the level of seriousness of the action is s divided by the amount of bystanders times $\alpha$.

Here, $\alpha$ is a parameter that determines the influence of the group; $\alpha$ is by default set to 0.5 . You adjust your opinion on the level of seriousness based on the nonintervention of the others"

\section{$\forall \mathrm{s}:$ REAL $\forall \mathrm{a}:$ ACTION $\forall \mathrm{n}: I N T E G E R$}

observe $(a) \wedge$ observe(no_intervention_by_others $) \wedge$ observe(number_of_bystanders(n)) $\wedge$

belief(has_seriousness $(\mathrm{a}, \mathrm{s})$ )

$\rightarrow \rightarrow$ belief(seriousness, $\mathrm{s} / \mathrm{n}^{\wedge} \alpha$ )

R7 "If you believe that the action has a level of seriousness of $\mathrm{x}$ and you believe that a certain norm has value $y$ and the action violates the norm $(x>y)$ then you believe that there is an emergency"

$\forall \mathrm{x}, \mathrm{y}:$ REAL

belief(seriousness, $x) \wedge$ belief(norm, $y) \wedge x>y \rightarrow \rightarrow$ belief(emergency) 
R8 "If you believe that there is an emergency then you have the desire to help"

$$
\text { belief(emergency) } \rightarrow \rightarrow \text { desire(i) }
$$

\section{Intention to help}

R9 "When you believe that you are personally responsible to help, and you have the desire to help then you have the intention to help"

desire(i) $\wedge$ belief(personal_responsibility) $\rightarrow \rightarrow$ intention(i)

\section{3) Opportunity to help}

R10 "If you believe that you are capable to help and have the required resources then you believe that you have the opportunity to help"

belief(capable(i)) $\wedge$ belief(resources(i) $\rightarrow \rightarrow$ belief(opportunity_for(i))

\section{4) Performance of Action}

R11 "The intention to help combined with the belief that you have the opportunity to help leads to the actual intervention"

$$
\begin{aligned}
& \text { intention(i) } \\
& \text { perform(i) belief(opportunity_for(i) }) \rightarrow \rightarrow
\end{aligned}
$$

\section{Step 3: Simulation}

To demonstrate the ability of the bystander model to generate interesting simulations, we have defined two different scenarios, inspired by the work from Chekroun and Brauer (2002). The first scenario involves low personal implication, and the second one involves high personal implication.

Low personal implication Chekroun and Brauer (2002) claim that a person's feeling of personal implication has an important impact on his or her incentive to intervene: if people do not feel personally implicated, their incentive to intervene is low. In areas like an elevator in a shopping mall, people usually feel low personal implication in case of norm violations, because shopping malls belong to a corporate business, and professional cleaning personnel are hired to keep the shopping mall clean.

Chekroun and Brauer performed an experiment (2002) in which a young man is waiting for the elevator. When people join him, he enters the elevator with them. Once the doors are closed, he takes out a marker and draws something on the wall of the elevator. For some time, he gives the other people the opportunity to react. After that, he leaves the elevator at the first floor. The results of this experiment indicated that the number of bystanders has an effect on the intervention rate. The more bystanders were present, the lower the probability that someone expressed disapproval.

High personal implication As opposed to the previous situation, people feel highly personally implicated in situations in which they feel it is their personal obligation to intervene. Chekroun and Brauer mention a park as an example of such a location. Maintenance of parks is usually paid via local taxes and thus by the inhabitants. Furthermore, aspects with respect to increasing consciousness about the environment play an important role in feeling personally implicated.

To test the intervention behavior and the effect of bystanders, the following experiment was performed by (Chekroun and Brauer 2002): two females are walking through the park. As soon as they approach other people, they start to drink from a plastic bottle and throw the bottle in the bushes. They make sure that all bystanders notice this. Then they walk away, and give the bystanders the opportunity to react to the littering event. In this experiment the number of people present did not affect the intervention behavior.

Simulation results We have used the situations described above to demonstrate our model's capability to replicate empirical findings. For each of the situations (low personal implication and high personal implication), we have run the simulation model for the situation with the amount of bystanders, the value of the norm and the level of seriousness as variables.

Figure 3 shows an example simulation trace. In this trace, time is on the horizontal axis and state properties are on the vertical axis. A dark box on top of a line indicates that the property is true during the corresponding time period. This trace shows a person's decision making process in a situation that is perceived as high personal implication.

As shown by Figure 3, at the start of the simulation (time point 0 ), the person observes an action: observe (action). This action is assumed to be the situation of littering in the park. Since this is a situation with high personal implication, the state property belief(has_seriousness (action)) has been assigned a value of 0.9. Together with a medium personal norm (value 0.5 on a $[0,1]$ scale), this leads to the belief that there is an emergency (at time point 2). Moreover, the number of bystanders is 3 , which leads to the belief that the costs of intervention are 3 and the audience inhibition is 3 (due to non-intervention of the bystanders), at time point 1 and 2 respectively. Next, because 3 is lower than the 'responsibility threshold' (see rule R5), which was set to 9 in this case, the person believes that he is personally responsible. Combined with the 


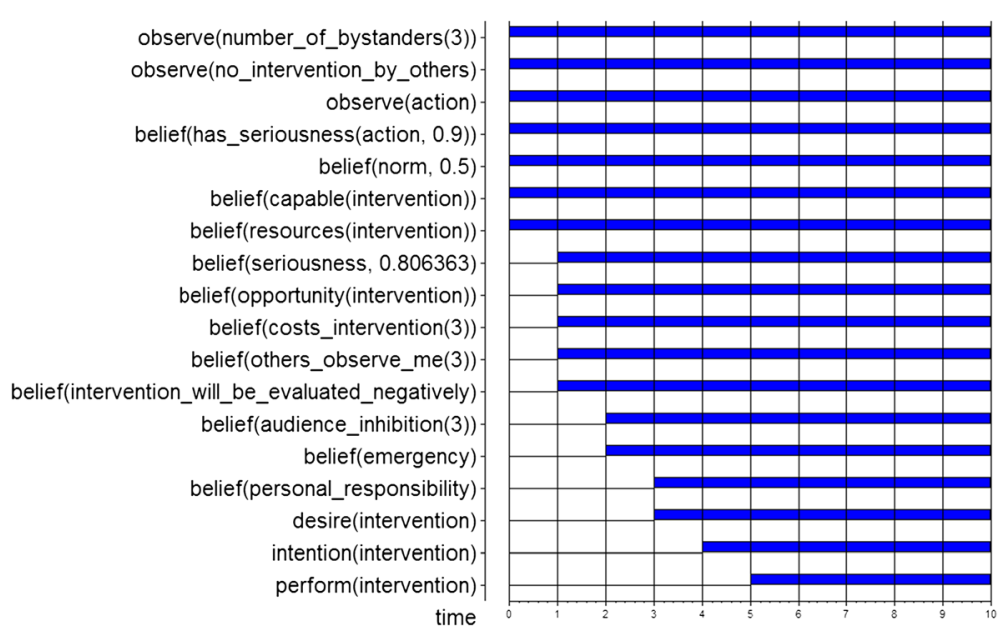

Figure 3 Simulation trace high personal implication with 3 bystanders.

desire to intervene, this belief leads to the intention to intervene. Meanwhile, the person also believes that he is capable to intervene and has the necessary resources for this, so he generates the belief that there is an opportunity to intervene. Finally, the intention and the opportunity together result in the actual intervention by the person (perform(intervention)) at time point 5 .

\section{Step 4: Evaluation}

The simulation described above is just one example of a pattern that can be produced by the model. Usually, to validate simulation models, large numbers of simulations are produced, and are compared to empirical data. To illustrate how this could be done for the bystander example, a number of additional simulation runs have been produced, using a variety of parameter settings. An overview of these settings and the corresponding results of the simulations is shown in Table 3.

In this table, the first column shows how many bystanders are present; this has been varied systematically between 1, 3, 4 and 5. The second column indicates the person's personal norm regarding the 'seriousness' of incidents. A norm with value 0.1 means that the person considers relatively mild events already emergency cases, and a norm of 0.8 means that events need to be very severe before (s)he considers them to be emergencies. Similarly, the seriousness value (column 3) determines how serious the observed event actually is (independent from the norm): 0.1 means that the observed event is not very serious, while an event rated with 0.9 is very serious. Next, the value for the thresholds (as used in rule $\mathrm{R} 5$ ) is mentioned in the fifth column.

The final three columns show the results of the simulations: they indicate, respectively, whether or not the agent feels personally responsible to intervene, has a desire to intervene and performs an intervention.
Roughly spoken, these results show that the model is able to reproduce the behaviour as found in the experiments performed by Chekroun and Brauer (2002). Intervention depends on the amount of bystanders and the personal commitment. The number of bystanders is important in the low personal implication scenarios (seriousness 0.1-0.5), while the bystanders do not have a large effect of the intervention behaviour is the high personal implication scenarios (seriousness 0.6-0.9).

\section{Strengths and weaknesses}

Although relatively simple, the example discussed in this chapter illustrates a number of benefits of agent-based modelling and simulation as a tool for criminological research. By formalising existing theories in terms of logical rules and executing these rules in a simulated environment, the effect of bystanders on intervention behaviour regarding norm violations could be studied for a wide variety of situations (e.g., 'what happens if person $\mathrm{P}$ is confronted with event $\mathrm{E}$, in a situation with $\mathrm{N}$ bystanders?'), without having to experiment with such situations in the real world. Note however that, when aiming to apply the model in a predictive manner, it would need to be 'tuned' by filling in critical values for a number of relevant parameters in such a way that these represent empirical data.

For the case of bystanders, the reader can probably, without many difficulties, appreciate the advantages of this approach over traditional methods, like the experiments described by Chekroun and Brauer (2002), for example. Performing such experiments involves a number of difficulties; among others, these experiments cost time, depend on the willingness and capabilities of the experimenter (e.g., one might need to hire actors to play the role of the 'litterers') and the possibilities to create the optimal circumstances (e.g., the weather, the amount 
Table 3 Overview of parameter settings and results of different simulations

\begin{tabular}{|c|c|c|c|c|c|c|}
\hline \# bystanders & Norm & Seriousness & Threshold & $\begin{array}{c}\text { Personal } \\
\text { responsibility }\end{array}$ & Desire & Intervention \\
\hline 1 & 0.1 & 0.2 & 2 & Yes & Yes & Yes \\
\hline 3 & 0.1 & 0.2 & 2 & No & Yes & No \\
\hline 4 & 0.1 & 0.2 & 2 & No & Yes & No \\
\hline 5 & 0.1 & 0.2 & 2 & No & Yes & No \\
\hline 1 & 0.1 & 0.5 & 5 & Yes & Yes & Yes \\
\hline 3 & 0.1 & 0.5 & 5 & Yes & Yes & Yes \\
\hline 4 & 0.1 & 0.5 & 5 & Yes & Yes & Yes \\
\hline 5 & 0.1 & 0.5 & 5 & No & Yes & Yes \\
\hline 1 & 0.1 & 0.9 & 9 & Yes & Yes & No \\
\hline 3 & 0.1 & 0.9 & 9 & Yes & Yes & Yes \\
\hline 4 & 0.1 & 0.9 & 9 & Yes & Yes & Yes \\
\hline 5 & 0.1 & 0.9 & 9 & Yes & Yes & Yes \\
\hline 1 & 0.5 & 0.1 & 1 & No & No & Yes \\
\hline 3 & 0.5 & 0.1 & 1 & No & No & No \\
\hline 4 & 0.5 & 0.1 & 1 & No & No & No \\
\hline 5 & 0.5 & 0.1 & 1 & No & No & No \\
\hline 1 & 0.5 & 0.5 & 5 & Yes & No & No \\
\hline 1 & 0.5 & 0.6 & 6 & Yes & Yes & No \\
\hline 1 & 0.5 & 0.9 & 9 & Yes & Yes & Yes \\
\hline 3 & 0.5 & 0.9 & 9 & Yes & Yes & Yes \\
\hline 4 & 0.5 & 0.9 & 9 & Yes & Yes & Yes \\
\hline 5 & 0.5 & 0.9 & 9 & Yes & Yes & Yes \\
\hline 1 & 0.8 & 0.9 & 9 & Yes & Yes & Yes \\
\hline 2 & 0.8 & 0.9 & 9 & Yes & Yes & Yes \\
\hline 3 & 0.8 & 0.9 & 9 & Yes & Yes & Yes \\
\hline 4 & 0.8 & 0.9 & 9 & Yes & No & No \\
\hline
\end{tabular}

of people present in the park), are not easy to repeat under the exact same conditions, and may lead to ethical objections. Instead, the simulated version of this experiment was quick and cheap, easy to manipulate and repeat, and less problematic on an ethical level. As a result, it allows researchers to perform systematic studies in which they predict, explain and compare an infinite amount of hypothetical scenarios without much effort.

On the other hand, also the downsides of modelling and simulation have probably become obvious: by definition, a model is a simplification of a real world system. As illustrated again by the bystander case study, models abstract away from many of the details of the real world, for the simple reason that it is impossible to include all factors at the smallest level of detail. For instance, the bystander model assumes that humans make perfect observations of the world around them, assumes that the decision to intervene can be represented via a simple threshold function, and does not include many parameters for individual personality characteristics. Although this does not have to be a problem (after all, an incomplete model can still lead to interesting new insights), it is important that the analyst is aware of these assumptions when drawing conclusions from simulation results. In particular, care should be taken when making claims that apply to more general cases than the scenarios studied with the model. Another potential weakness of simulation models in general is that they satisfy the property of equifinality, i.e., one particular simulation outcome (e.g., the fact that the model predicts intervention by bystanders) can be generated by a range of different micro-interactions. Hence, the mechanisms that resulted in a particular outcome should not be interpreted as the only possible condition for this result.

\section{Anticipated future developments}

Agent-based modelling and simulation is a large, interdisciplinary area of research, with a virtually infinite 
amount of application domains, including the social sciences, economics, and earth and life sciences. Also within Criminology, the use of simulations to explain and predict various aspects of the complex dynamics of crime is still in its infancy. In Gerritsen (2010), a number of different examples are presented, such as the study of violent behaviour, social learning of juvenile delinquency, and the spatio-temporal dynamics of burglary. For the future, the approach has the potential to tackle an even larger number of domains. A particularly interesting class of problems is those cases where changes in the current policy are suggested, for instance regarding the influence of the male-female ratio among guardians on the prisoners' behaviour, planning of novel residential areas, installation of security camera's, and the composition of school classes. Moreover, the rapid developments in the ICT industry, resulting in improved possibilities to obtain large amounts of data, as well as an ever-increasing processing power of computing devices, make it easier to validate and fine-tune simulation models based on empirical data. Hence, we have probably only seen the tip of the iceberg when it comes to the use of computational modelling and simulation for criminological purposes.

\section{Suggestions for further reading}

P.H.M. van Baal (2004), Computer Simulations of Criminal Deterrence: from Public Policy to Local Interaction to Individual Behaviour. Ph.D. Thesis, Erasmus University Rotterdam. Boom Juridische Uitgevers.

The approach presented in this thesis specifically aims at simulating the process of deterrence. The main aim of the work is to investigate how the probability of being punished influences the amount of crime. A simulation model is presented where each potential offender is part of a social network that consists of several agents. All agents repeatedly face a choice between rule compliance and rule transgression. If agents transgress, they have a probability of being audited and punished.

P.L. Brantingham, U. Glässer, K. Singh, and M. Vajihollahi (2005). Mastermind: Modeling and Simulation of Criminal Activity in Urban Environments. Technical Report SFU-CMPTTR-2005-01, Simon Fraser University.

In this article an approach to analyse the spatiotemporal dynamics of crime is presented. The approach is based on a Distributed Abstract State Machine (DASM) formalism, combined with a multi-agent based modelling paradigm. The agents involved are capable of learning using a form of behavioural reinforcement learning, where based on past experiences certain preferences are developed that may influence future choices.
C. Gerritsen (2010), Caught in the Act: Investigating Crime by Agent-Based Simulation

In her Ph.D. thesis Gerritsen provides an overview of the possibilities of applying techniques from the domain of Artificial Intelligence to different criminological domains (i.e., biological and cognitive aspects of criminal behavior; dynamics of delinquent behavior among adolescents; spatio-temporal dynamics of crime). For each of the domains it is illustrated how AI techniques can be beneficial in understanding, analyzing or predicting criminal behavior.

L. Liu and J. Eck (eds.) (2008), Artificial Crime Analysis Systems: Using Computer Simulations and Geographic Information Systems Information Science Reference: Hershey, PA, USA

In this volume leading research on the use of computer simulation of crime patterns are discussed to reveal hidden processes of urban crimes. Criminology, computer simulation and geographic information systems are combined in this interdisciplinary approach.

L. Liu, X. Wang, J. Eck., and J. Liang (2005). Simulating Crime Events and Crime Patterns in RA/CA Model. In F. Wang (ed.), Geographic Information Systems and Crime Analysis. Singapore: Idea Group.

The possibility of simulating individual crime events in order to generate plausible crime patterns is explored. This approach is based on a Cellular Automaton (CA), in which the main elements are offenders, targets, and crime places. Different attributes of the model can be manipulated, among which motivation of offenders, capability of guardians, and accessibility of places.

D. Reis, A. Melo, A.L.V. Coelho, and V. Furtado (2006), Towards Optimal Police Patrol Routes with Genetic Algorithms. In: Mehrotra, S., et al. (eds.), ISI 2006. LNCS 3975.

In this paper the authors use a tool to investigate the influence that different police control routes have on the reduction of crime rates. The approach comprises an artificial society consisting of various agents, in particular criminals and policemen. The first results are presented that were achieved with GAPatrol, an evolutionary multi agent-based simulation tool devised to assist police managers in the design of effective police patrol route strategies.

\section{Endnotes}

The theoretical part of the chapter previously appeared as: Gerritsen, C. and Klein, M.C.A. (2014), Dynamical Simulation as a Research Tool in Criminology. In: Bruinsma, G. and Weisburd, D. (eds). Encyclopedia of Criminology and Criminal Justice. New York: Springer Verlag, pp. 1220-1231. 
The case study appeared as: (Gerritsen 2011). The Bystander Effect: Agent-Based Simulation of People's Reaction to Norm Violation. In: Lu, B., Zhang, L., and Kwok, J. (eds), Proceedings of the $18^{\text {th }}$ International Conference on Neural Information Processing, ICONIP'11, Lecture Notes in Artificial Intelligence, Springer Verlag, pp. 26-35.

\section{Competing interests}

The author declares that she has no competing interests.

Received: 13 December 2014 Accepted: 13 December 2014 Published online: 12 February 2015

\section{References}

Baal, PHM van (2004). Computer Simulations of Criminal Deterrence: from Public Policy to Local Interaction to Individual Behaviour. Ph.D. Thesis. Rotterdam, The Netherlands: Erasmus University Rotterdam. Boom Juridische Uitgevers.

Banos, A, Lang, C, Marilleau, N (2015). Agent-Based Spatial Simulation with NetLogo: Introduction and Bases, Wiley-ISTE, to appear.

Bellifemine, FL, Caire, G and Greenwood, D (2007). Developing Multi-Agent Systems with JADE, Hoboken, USA: Wiley \& sons.

Bosse, T and Gerritsen, C (2010). Social Simulation and Analysis of the Dynamics of Criminal Hot Spots. In Journal of Artificial Societies and Social Simulation, vol. 13, issue 2. http://jasss.soc.surrey.ac.uk/13/2/5.html.

Bosse, T, Jonker, CM, van der Meij, L, \& Treur, J. (2007). A Language and Environment for Analysis of Dynamics by Simulation. International Journal of Artificial Intelligence Tools, Singapore: World Scientific. 16(3), 435-464.

Bosse, T, Gerritsen, C, Hoogendoorn, M, Jaffry, SW, and Treur, J. (2011). AgentBased versus Population-Based Simulation of Displacement of Crime: a Comparative Study. In: Web Intelligence and Agent-Systems: An International Journal, vol. 9. Amsterdam, The Netherlands: IOS Press, (pp. 147-160).

Brantingham, PL, Glässer, U, Singh, K, Vajihollahi, M (2005). Mastermind: Modeling and Simulation of Criminal Activity in Urban Environments. Technical Report SFU-CMPTTR-2005-01, Vancouver, Canada: Simon Fraser University.

Chekroun, P, \& Brauer, M. (2002). The bystander effect and social control behaviour: the effect of the presence of others on people's reactions to norm violations. European Journal of Social Psychology, 32, 853-867.

Elffers, H, \& van Baal, P. (2008). Spatial Backcloth is not that important in simulation research: An illustration from simulating perceptual deterrence. In L Liu \& JE Eck (Eds.), Artificial crime analysis systems (pp. 19-34). Hershey, PA: IGl Global.

Freeman, S, Walker, M, Bordon, R, \& Latané, B. (1975). Diffusion of responsibility and restaurant tipping: cheaper by the bunch. Personality and Social Psychology Bulletin, 1, 584-587.

Gerritsen, C (2010). Caught in the Act: investigating crime by agent-based simulation. Ph.D. Thesis. Amsterdam, The Netherlands: VU University Amsterdam.

Gerritsen, C. (2011). The Bystander Effect: Agent-Based Simulation of People's Reaction to Norm Violation. In: Lu, B., Zhang, L., and Kwok, J. (eds), Proceedings of the $18^{\text {th }}$ International Conference on Neural Information Processing, ICONIP'11: Lecture Notes in Artificial Intelligence, Springer Verlag, (pp. 26-35)

Gerritsen, C, Bosse, T (2015). Crime Prevention: a Model-Based Reasoning Approach. In Magnani, L. and Bertolotti, T. (Eds.), Springer handbook of Model-based Science, to appear. Berlin, Germany: Springer Verlag.

Gilbert, N. (2008). Agent-based Models. London: Sage Publications.

Groff, ER (2005). The Geography of Juvenile Crime Place Trajectories. Ph.D. Thesis: College Park, Maryland, USA: University of Maryland.

Hayslett-McCall, K, Qui, F, Curtin, KM, Chastain, B, Schubert, J, and Carver, V. (2008). The Simulation of the journey to residential burglary. In Liu, L and Eck, J. (Eds.), Artificial Crime Analysis Systems: using computer simulations and geographic information systems, Hershey, PA, USA: Information Science Reference, (pp. 281-300).

Latané, B, \& Darley, JM. (1968). Group inhibition of bystander intervention in emergencies. Journal of Personality and Social Psychology, 10, 215-221.

Latané, B, \& Nida, S. (1981). Ten years of research on group size and helping. Psychological Bulletin, 89, 308-324.

Levy, P, Lundgren, D, Ansel, M, Fell, D, Fink, B, \& McGrath, JE. (1972). Bystander effect in a demand-without-threat situation. Journal of Personality and Social Psychology, 24, 166-171.
Liu, L, \& Eck, J (Eds.). (2008). Artificial Crime Analysis Systems: Using Computer Simulations and Geographic Information Systems Information. Hershey, PA, USA: Science Reference.

Liu, L, Wang, X, Eck, J, \& Liang, J. (2005). Simulating Crime Events and Crime Patterns in RA/CA Model. In F Wang (Ed.), Geographic Information Systems and Crime Analysis (pp. 197-213). Singapore: Idea Group.

Luke, S, Cioffi-Revilla, C, Panait, L, Sullivan, K, \& Balan, G. (2005). MASON: a multiagent simulation environment. Simulation: Transactions of the society for modelling and simulation international, 82(7), 517-527.

Malleson, N and Brantingham, P (2008). Prototype Burglary Simulations for Crime Reduction and Forecasting. In Crime Patterns and Analysis, vol. 2, Little Rock, Arkansas, USA. issue 1, 2008, (pp. 47-66).

Nikolai, C, \& Madey, G. (2009). Tools of the Trade: A Survey of Various AgentBased Modeling Platforms. Journal of Artificial Societies and Social Simulations, 12(2), 2.

Rao, AS \& Georgeff, MP (1991). Modelling rational agents within a BDIarchitecture. In Allen, J et al. (Eds), Proceedings of the Second International Conference on Principles of Knowledge Representation and Reasoning, (KR'91). San Francisco, USA: Morgan Kaufmann. (pp. 473-484).

Reis, D, Melo, A, Coelho, ALV, and Furtado, V (2006). Towards Optimal Police Patrol Routes with Genetic Algorithms. In Mehrotra, S, et al. (Eds.), ISI 2006: LNCS 3975, Springer, Berlin Heidelberg. (pp. 485-491).

Shannon, RF (1975). Systems simulation. Upper Saddle River, NJ, USA: Prentice-Hall.

\section{Submit your manuscript to a SpringerOpen ${ }^{\circ}$ journal and benefit from:}

- Convenient online submission

Rigorous peer review

- Immediate publication on acceptance

- Open access: articles freely available online

- High visibility within the field

- Retaining the copyright to your article

Submit your next manuscript at springeropen.com 